Provided for non-commercial research and education use. Not for reproduction, distribution or commercial use.

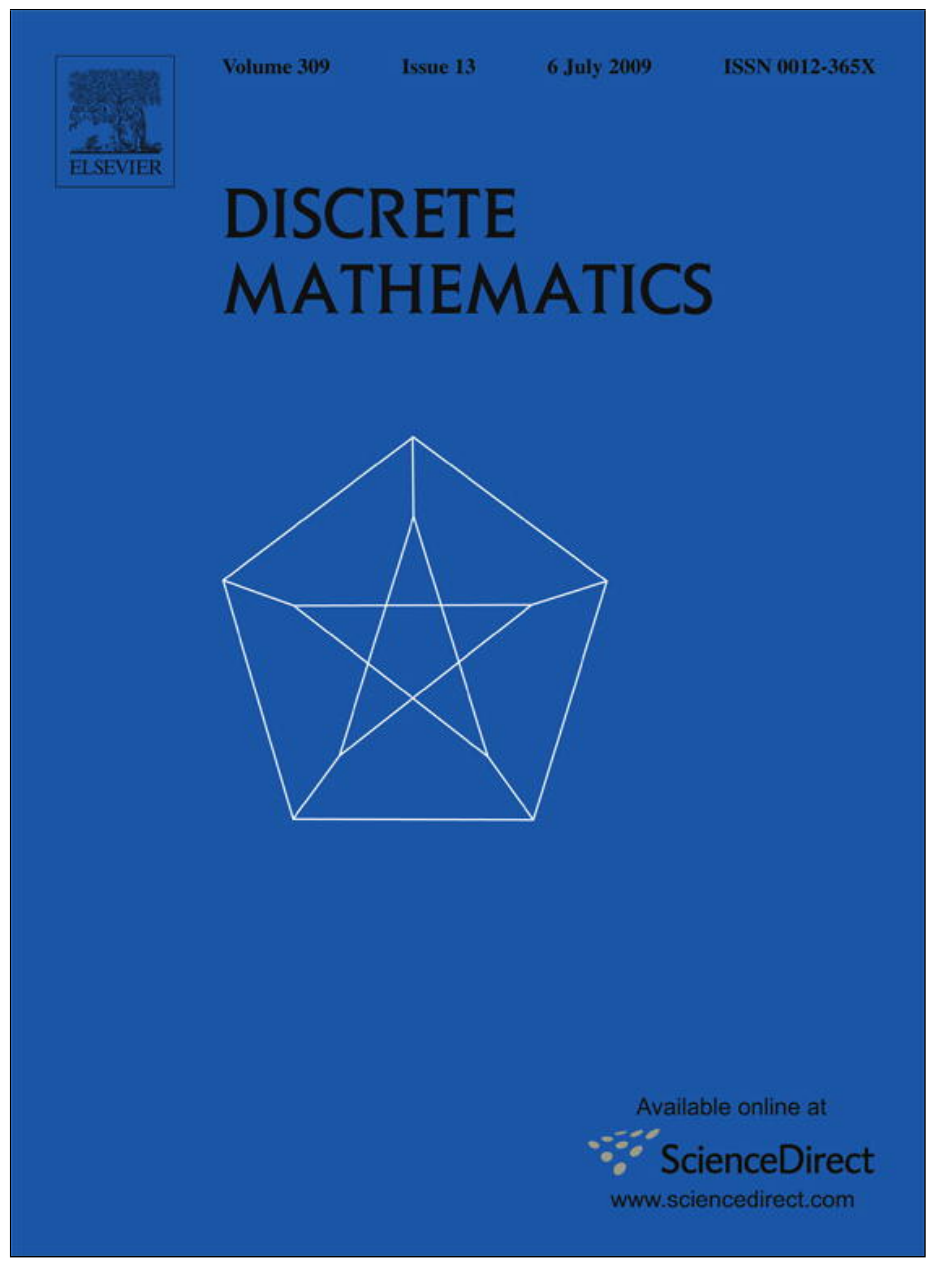

This article appeared in a journal published by Elsevier. The attached copy is furnished to the author for internal non-commercial research and education use, including for instruction at the authors institution and sharing with colleagues.

Other uses, including reproduction and distribution, or selling or licensing copies, or posting to personal, institutional or third party websites are prohibited.

In most cases authors are permitted to post their version of the article (e.g. in Word or Tex form) to their personal website or institutional repository. Authors requiring further information regarding Elsevier's archiving and manuscript policies are encouraged to visit:

http://www.elsevier.com/copyright 


\title{
Stability of the path-path Ramsey number
}

\author{
András Gyárfás ${ }^{\mathrm{a}}$, Gábor N. Sárközy ${ }^{\mathrm{a}, \mathrm{b}, *}$, Endre Szemerédi ${ }^{\mathrm{c}, \mathrm{d}}$ \\ ${ }^{a}$ Computer and Automation Research Institute, Hungarian Academy of Sciences, Budapest, P.O. Box 63, Budapest, H-1518, Hungary \\ ${ }^{\mathrm{b}}$ Computer Science Department, Worcester Polytechnic Institute, Worcester, MA, 01609, USA

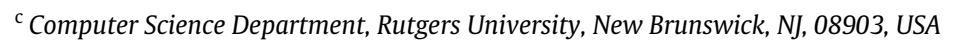 \\ d Institute for Advanced Study, Princeton, NJ, 08540, USA
}

\section{A R T I C L E I N F O}

\section{Article history:}

Received 18 August 2008

Received in revised form 15 February 2009

Accepted 18 February 2009

Available online 20 March 2009

\section{Keywords:}

Ramsey theory

Stability

Path

\begin{abstract}
A B S T R A C T
Here we prove a stability version of a Ramsey-type Theorem for paths. Thus in any 2-coloring of the edges of the complete graph $K_{n}$ we can either find a monochromatic path substantially longer than $2 n / 3$, or the coloring is close to the extremal coloring.
\end{abstract}

(C) 2009 Elsevier B.V. All rights reserved.

\section{Introduction}

The vertex-set and the edge-set of the graph $G$ is denoted by $V(G)$ and $E(G) . K_{n}$ is the complete graph on $n$ vertices, $K_{r+1}(t)$ is the complete $(r+1)$-partite graph where each class contains $t$ vertices and $K_{2}(t)=K(t, t)$ is the complete bipartite graph between two vertex classes of size $t$. We denote by $(A, B, E)$ a bipartite graph $G=(V, E)$, where $V=A+B$, and $E \subset A \times B$. For a graph $G$ and a subset $U$ of its vertices, $\left.G\right|_{U}$ is the restriction of $G$ to $U$. The set of neighbors of $v \in V$ is $N(v)$. Hence the size of $N(v)$ is $|N(v)|=\operatorname{deg}(v)=\operatorname{deg}_{G}(v)$, the degree of $v$. The minimum degree is denoted by $\delta(G)$ and the maximum degree by $\Delta(G)$ in a graph $G$. When $A, B$ are subsets of $V(G)$, we denote by $e(A, B)$ the number of edges of $G$ with one endpoint in $A$ and the other in $B$. In particular, we write $\operatorname{deg}(v, U)=e(\{v\}, U)$ for the number of edges from $v$ to $U$. A graph $G_{n}$ on $n$ vertices is $\gamma$-dense if it has at least $\gamma\left(\begin{array}{c}n \\ 2\end{array}\right)$ edges. A bipartite graph $G(k, l)$ is $\gamma$-dense if it contains at least $\gamma k l$ edges.

For graphs $G_{1}, G_{2}, \ldots, G_{r}$, the Ramsey number $R\left(G_{1}, G_{2}, \ldots, G_{r}\right)$ is the smallest positive integer $n$ such that if the edges of a complete graph $K_{n}$ are partitioned into $r$ disjoint color classes giving $r$ graphs $H_{1}, H_{2}, \ldots, H_{r}$, then at least one $H_{i}(1 \leq i \leq r)$ has a subgraph isomorphic to $G_{i}$. The existence of such a positive integer is guaranteed by Ramsey's classical result [12]. The number $R\left(G_{1}, G_{2}, \ldots, G_{r}\right)$ is called the Ramsey number for the graphs $G_{1}, G_{2}, \ldots, G_{r}$. There is very little known about $R\left(G_{1}, G_{2}, \ldots, G_{r}\right)$ even for very special graphs (see e.g. [4] or [11]). For $r=2$ a theorem of Gerencsér and Gyárfás [3] states that

$$
R\left(P_{n}, P_{n}\right)=\left\lfloor\frac{3 n-2}{2}\right\rfloor
$$

In this paper we prove a stability version of this theorem. Since this is what we needed in a recent application [6], actually we prove the result in a slightly more general context; we work with 2-edge multicolorings $\left(G_{1}, G_{2}\right)$ of a graph $G$. Here

\footnotetext{
* Corresponding author at: Computer Science Department, Worcester Polytechnic Institute, Worcester, MA, 01609, USA.

E-mail addresses: gyarfas@sztaki.hu (A. Gyárfás), gsarkozy@cs.wpi.edu (G.N. Sárközy), szemered@cs.rutgers.edu (E. Szemerédi).
} 
multicoloring means that the edges can receive more than one color, i.e. the graphs $G_{i}$ are not necessarily edge disjoint. The subgraph colored with color $i$ only is denoted by $G_{i}^{*}$, i.e.

$$
G_{1}^{*}=G_{1} \backslash G_{2}, \quad G_{2}^{*}=G_{2} \backslash G_{1} .
$$

In order to state the theorem we need to define a relaxed version of the extremal coloring for (1).

Extremal Coloring (with parameter $\alpha$ ): There exists a partition $V(G)=A \cup B$ such that

- $|A| \geq(2 / 3-\alpha)|V(G)|,|B| \geq(1 / 3-\alpha)|V(G)|$.

- The graph $\left.G_{1}^{*}\right|_{A}$ is $(1-\alpha)$-dense and the bipartite graph $\left.G_{2}^{*}\right|_{A \times B}$ is $(1-\alpha)$-dense. (Note that we have no restriction on the coloring inside the smaller set.)

Then the following stability version of the Gerencsér-Gyárfás Theorem claims that we can either find a monochromatic path substantially longer than $2 n / 3$, or the coloring is close to the extremal coloring.

Theorem 1.1. For every $\alpha>0$ there exists a positive real $\eta(0<\eta \ll \alpha \ll 1$ where $\ll$ means sufficiently smaller $)$ and $a$ positive integer $n_{0}$ such that for every $n \geq n_{0}$ the following holds: if the edges of the complete graph $K_{n}$ are 2-multicolored then we have one of the following two cases.

- Case 1: $K_{n}$ contains a monochromatic path $P$ of length at least $\left(\frac{2}{3}+\eta\right) n$.

- Case 2: This is an Extremal Coloring (EC) with parameter $\alpha$.

We remark that while for some classical density results the corresponding stability versions are well-known (see [1]), stability questions in Ramsey problems only emerged recently (see [5,7,10]).

\section{Tools}

Theorem 1.1 can also be proved from the Regularity Lemma [13], however, here we use a more elementary approach using only the Kővári-Sós-Turán bound [8]. This is part of a new direction to "de-regularize" some proofs, namely to replace the Regularity Lemma with more elementary classical extremal graph theoretic results such as the Kővári-Sós-Turán bound (see e.g. [9]).

Lemma 2.1 (Theorem 3.1 on page 328 in [1]). There is an absolute constant $\beta>0$ such that if $0<\epsilon<1 / r$ and we have a graph $G$ with

$$
|E(G)| \geq\left(1-\frac{1}{r}+\epsilon\right) \frac{n^{2}}{2}
$$

then $G$ contains a $K_{r+1}(t)$, where

$$
t=\left\lfloor\frac{\beta \log n}{r \log 1 / \epsilon}\right\rfloor \text {. }
$$

For $r=1$ this is essentially the Kővári-Sós-Turán bound [8] and for general $r$ this was proved by Bollobás, Erdős and Simonovits [2]. Here we will use the result only for $r=1$.

\section{Outline of the proof}

We will need the following definition. Given a graph $G$ and a positive integer $k$, we say that a subset $W$ of the vertex set $V(G)$ is $k$-well-connected if for any two vertices $u, v \in W$ there are at least $k$ internally vertex disjoint paths of length at most three connecting $u$ and $v$ in $G$ (note that these paths might leave $W$ ). We will use this definition with $k=\eta n$, in this case we just say shortly that $W$ is well-connected.

We will follow a similar outline as in applications of the Regularity Lemma. However, a regular pair will be replaced with a complete balanced bipartite graph $K(t, t)$ with $t \geq c \log n$ for some constant $c$ (thus the size of the pair is somewhat smaller but this is still good enough for our purposes). Then a monochromatic connected matching in the reduced graph (the usual tool in these types of proofs using the Regularity Lemma) will be replaced with a set of vertex disjoint monochromatic complete balanced bipartite graphs $K_{i}\left(t_{i}, t_{i}\right), 1 \leq i \leq s$ with $t_{i} \geq c \log n, 1 \leq i \leq s$ for some constant $c$. Moreover, these bipartite graphs are all contained in a set $W$ that is well-connected in this color. Let us call a set of bipartite graphs like this a monochromatic well-connected complete balanced bipartite graph cover (we are covering vertices here). The size of this cover is the total number of vertices in the union of these complete bipartite graphs.

Then Theorem 1.1 will follow from the following lemma.

Lemma 3.1. For every $\alpha>0$ there exist a positive real $\eta(0<\eta \ll \alpha \ll 1$ where $\ll$ means sufficiently smaller $)$ and a positive integer $n_{0}$ such that for every $n \geq n_{0}$ the following holds: if the edges of the complete graph $K_{n}$ are 2-multicolored then we have one of the following two cases.

- Case 1: $K_{n}$ contains a monochromatic well-connected complete balanced bipartite graph cover of size at least $\left(\frac{2}{3}+2 \eta\right) n$.

- Case 2: This is an Extremal Coloring (EC) with parameter $\alpha$. 
Indeed, let us assume that we have Case 1 in this Lemma. Denote the two color classes of $K_{i}\left(t_{i}, t_{i}\right)$ by $V_{1}^{i}$ and $V_{2}^{i}$ for $1 \leq i \leq s$. Since this cover is inside the same set $W$ that is well-connected in this color (say red), we can find a red connecting path $P_{1}$ of length at most 3 from an arbitrary vertex of $V_{2}^{1}$ to an arbitrary vertex of $V_{1}^{2}$. Similarly we can find a red connecting path $P_{2}$ of length at most 3 from a vertex of $V_{2}^{2}$ to a vertex of $V_{1}^{3}$ that is vertex disjoint from $P_{1}$. We continue in this fashion until we find a red connecting path $P_{s-1}$ of length at most 3 from a vertex of $V_{2}^{s-1}$ to a vertex of $V_{1}^{s}$ that is vertex disjoint from each of the connecting paths $P_{i}, 1 \leq i \leq s-2$, constructed so far. Furthermore, we can also guarantee that in this connecting process from any $V_{j}^{i}, 1 \leq j \leq 2,1 \leq \bar{i} \leq s$ we never use up more than $\eta\left|V_{j}^{i}\right|$ vertices. Indeed, then during the whole process the total number of forbidden vertices is at most

$$
\frac{4 s}{\eta} \leq \frac{4 n}{2 c \eta \log n} \ll \eta n,
$$

(if $n$ is sufficiently large) and thus we can always select the next connecting path that is vertex disjoint from the ones constructed so far.

We remove the internal vertices of these connecting paths $P_{i}$ from the complete balanced bipartite graphs $K_{i}\left(t_{i}, t_{i}\right)$. By doing this we may create some discrepancies in the cardinalities of the two color classes. We remove some additional vertices to assure that now we have the same number $t_{i}^{\prime}\left(\geq(1-\eta) t_{i}\right)$ of vertices left in both color classes. Now we can connect the endpoint of $P_{i-1}$ in $V_{1}^{i}$ and the endpoint of $P_{i}$ in $V_{2}^{i}$ by a red Hamiltonian path $Q_{i}$ in the remainder of $K_{i}\left(t_{i}, t_{i}\right)$ (using the fact that this is a balanced complete bipartite graph). Putting together the connecting paths $P_{i}$ and these Hamiltonian paths $Q_{i}$ we get a red path $P$ of length at least

$$
\left(\frac{2}{3}+2 \eta-\frac{2}{3} \eta\right) n \geq\left(\frac{2}{3}+\eta\right) n
$$

Actually, in the application [6] we needed a slightly stronger statement, namely that for each vertex of $P$ we have at least (c/2) $\log n$ choices, but this is a straightforward corollary of the proof.

Corollary 3.2. In Case 1 of Theorem 1.1, in the process of finding $P$, for each vertex of the path $P$ we have at least $c_{1} \log n$ choices for some constant $c_{1}$.

\section{Monochromatic well-connected components}

In this section we show that in any 2-multicoloring of $K_{n}$ there is a large set $W$ and a color such that $W$ is $k$-well-connected in this color.

Lemma 4.1. For every integer $k$ and for every 2-multicolored $K_{n}$ there exist $W \subset V\left(K_{n}\right)$ and a color (say color 1 ) such that $|W| \geq n-28 k$ and $W$ is $k$-well-connected in the color 1 subgraph of $K_{n}$.

Proof. Assume that a 2-multicoloring is given on $K_{n}$ - in fact it is enough to prove the lemma for a coloring obtained by ignoring one of the colors of every 2-colored edge. A pair $u, v \in V\left(K_{n}\right)$ is bad for color 1 if there are no $k$ internally vertex disjoint paths of length at most three from $u$ to $v$ all monochromatic in color 1 . Let $m$ be the maximum number of vertex disjoint bad pairs for color 1 . If $m<2 k$ then deleting $2 m<4 k$ vertices of a maximum matching of bad pairs for color 1 , we have a set $W$ of more than $n-4 k$ vertices that is $k$-well-connected for color 1 and the proof is finished (with $24 k$ to spare). Note that deleting these $2 m$ vertices will not destroy the short connecting paths between the remaining pairs of vertices, since these paths are allowed to leave $W$, so they may use the deleted vertices.

Otherwise select a matching $\left\{u_{1} v_{1}, \ldots, u_{2 k} v_{2 k}\right\}$ of $2 k$ bad pairs for color 1 . For $t \in[2 k], i, j \in[1,2]$ define $A(t, i, j)$ as the set of vertices adjacent to $u_{t}$ in color $i$ and to $v_{t}$ in color $j$. From the definition of bad pairs $|A(t, 1,1)|<k$. For the same reason - using König's theorem - all edges of color 1 in the bipartite graph $[A(t, 1,2), A(t, 2,1)]$ can be met by a set $S_{t}$ of less than $k$ vertices. Set $B_{t}=A(t, 1,2) \backslash S_{t}, C_{t}=A(t, 2,1) \backslash S_{t}$. Observe that between $B_{t}$ and $C_{t}$ we have a complete bipartite graph in color 2 (if both are non-empty). A set $B_{t}\left(C_{t}\right)$ is called small if it has less than $2 k$ elements. Define $H_{t}$ as the union of $A(t, 1,1) \cup S_{t}$ and the small sets $B_{t}, C_{t}$ (that is, we include $B_{t}$ or $C_{t}$ only when the set is small). Observe that $\left|H_{t}\right|<6 k$. Consider the hypergraph $\mathscr{H}$ with vertex set $X=V\left(K_{n}\right) \backslash\left(\cup_{t \in[2 k]}\left\{u_{t}, v_{t}\right\}\right)$ and edge set $X \cap H_{t}$ for $t \in[2 k]$. Let $M$ be the set of vertices of $\mathscr{H}$ with degree at least $k / 2$. Then

$$
12 k^{2}=2 k \times 6 k \geq \sum_{t=1}^{2 k}\left|H_{t}\right|=\sum_{x \in X} \operatorname{deg}(x) \geq \sum_{x \in M} \operatorname{deg}(x) \geq|M|(k / 2)
$$

implying that $24 k \geq|M|$. Therefore $|X \backslash M| \geq n-24 k-4 k=n-28 k$. We claim that $W=X \backslash M$ is $k$-well-connected in color 2 and that will finish the proof.

To prove the claim, consider a pair of vertices $x, y \in W$. From the definition of $W, x, y$ are both in less than $k / 2$ edges of $\mathcal{H}$. Thus $x, y$ are both covered by at least $k$ sets in the form $Y_{t}=\left(\cup_{i, j \in[1,2]} A(t, i, j)\right) \backslash H_{t}$ where $t \in[2 k]$. Therefore in every $Y_{t}$ the pair $x, y$ can be connected in color 2 either by a path of length two through $u_{t}$ (if $x, y \in A(t, 2,2) \cup A(t, 2,1)$ ) or through $v_{t}$ (if $x, y \in A(t, 2,2) \cup A(t, 1,2)$ ) or through a path of length three (if one of $x, y$ is in $A(t, 2,1)$ and the other is in $A(t, 1,2$ ). 
It is easy to see-using that there are at least $k$ choices for $t,\left|B_{t}\right|=\left|A(t, 1,2) \backslash S_{t}\right| \geq 2 k,\left|C_{t}\right|=\left|A(t, 2,1) \backslash S_{t}\right| \geq 2 k$ and that we have a complete bipartite graph between $B_{t}$ and $C_{t}$ in color 2 -that there are at least $k$ internally edge disjoint paths of length at most three in color 2 connecting $x, y$.

\section{Proof of Lemma 3.1}

Assume that we have an arbitrary 2-multicoloring (red/blue) of $K_{n}$. We shall assume that $n$ is sufficiently large and use the following main parameters

$$
0 \ll \eta \ll \alpha \ll 1,
$$

where $a \ll b$ means that $a$ is sufficiently small compared to $b$. In order to present the results transparently we do not compute the actual dependencies, although it could be done. We will use the constant

$$
c=\left(\frac{\eta}{2}\right)^{\frac{8}{\eta^{3}}} \frac{\beta}{2 \log \frac{1}{\eta}},
$$

where $\beta$ is from Lemma 2.1 .

Let us apply Lemma 4.1 with $k=\eta n$ to find a $W \subset V\left(K_{n}\right)$ and a color (say red) such that $|W| \geq(1-28 \eta) n$ and $W$ is $\eta n$-well-connected (or shortly, well-connected) in the red subgraph of $K_{n}$. Put $R=K_{n} \backslash W$, then we have $|R| \leq 28 \eta n$. From now on we will work inside $W$.

We may assume that inside $W$ the red density is at least $\eta$, since otherwise we can switch colors as the blue-only subgraph is almost complete. Thus we can apply Lemma 2.1 with $r=1$ and $\epsilon=\eta$ to the red subgraph inside $W$ to find a red complete balanced bipartite subgraph $K_{1}\left(t_{1}, t_{1}\right)$ in $W$ with

$$
t_{1}=\frac{\beta}{2 \log \frac{1}{\eta}} \log n
$$

(for simplicity we assume that this is an integer). We remove this $K_{1}\left(t_{1}, t_{1}\right)$ from $W$ and in the remainder of $W$ iteratively we find red complete balanced bipartite graphs $K\left(t_{1}, t_{1}\right)$ while we can. Suppose that we found this way the red well-connected complete balanced bipartite graph cover

$$
M_{1}=\left(K_{1}\left(t_{1}, t_{1}\right), K_{2}\left(t_{1}, t_{1}\right), \ldots, K_{s_{1}}\left(t_{1}, t_{1}\right)\right)
$$

for some positive integer $s_{1}$. If this red cover $M_{1}$ has size $\left|M_{1}\right| \geq(2 / 3+2 \eta) n$, then we are done, we have Case 1 in Lemma 3.1 . Otherwise we will show that we can either increase the size of this red cover by an $\eta^{2} / 2$-fraction, or we can find directly a monochromatic well-connected complete bipartite graph cover of size at least $(2 / 3+2 \eta) n$ unless we are in the Extremal Coloring (Case 2), as desired.

We know that at least we have

$$
\left|M_{1}\right| \geq \frac{\eta}{4} n
$$

since otherwise in the remainder of $W$ the red density is still at least $\eta / 2$, and we can still apply Lemma 2.1 in the remainder to find a red $K\left(t_{1}, t_{1}\right)$. Let $K_{i}\left(t_{1}, t_{1}\right)=\left(V_{1}^{i}, V_{2}^{i}\right), 1 \leq i \leq s_{1}$. Denote

$$
V_{1}=\bigcup_{i=1}^{s_{1}} V_{1}^{i}, \quad V_{2}=\bigcup_{i=1}^{s_{1}} V_{2}^{i} \quad \text { and } \quad V_{3}=W \backslash\left(V_{1} \cup V_{2}\right) \text {. }
$$

From

$$
\left|V_{1}\right|+\left|V_{2}\right|<\left(\frac{2}{3}+2 \eta\right) n
$$

we have

$$
\left|V_{3}\right|>\left(\frac{1}{3}-30 \eta\right) n
$$

Furthermore, since in $V_{3}$ we cannot pick another red complete balanced bipartite subgraph $K\left(t_{1}, t_{1}\right)$, by Lemma $2.1 V_{3}$ is $(1-\eta)$-dense in the blue-only subgraph.

Next let us look at the bipartite graphs $\left(V_{1}, V_{3}\right)$ and $\left(V_{2}, V_{3}\right)$. We will show that either one of them is $(1-2 \eta)$-dense in blue-only or we can increase our red cover $M_{1}$. Indeed, assume first the following:

(i) There is a subcover of $M_{1}$

$$
M_{1}^{\prime}=\left(K_{i_{1}}\left(t_{1}, t_{1}\right), K_{i_{2}}\left(t_{1}, t_{1}\right), \ldots, K_{s_{1}^{\prime}}\left(t_{1}, t_{1}\right)\right)
$$

with $1 \leq s_{1}^{\prime} \leq s_{1}$ such that if we denote $V_{1}^{\prime}=\cup_{j=1}^{s_{1}^{\prime}} V_{1}^{i_{j}}, V_{2}^{\prime}=\cup_{j=1}^{s_{1}^{\prime}} V_{2}^{i_{j}}$ we have

- $\left|V_{1}^{\prime}\right|=\left|V_{2}^{\prime}\right| \geq \eta\left|V_{1}\right|=\eta\left|V_{2}\right|$, and

- the bipartite graphs $\left(V_{1}^{i_{j}}, V_{3}\right)$ and $\left(V_{2}^{i_{j}}, V_{3}\right)$ are both $\eta$-dense in red for every $1 \leq j \leq s_{1}^{\prime}$. 
Consider the bipartite graph $\left(V_{1}^{i_{j}}, V_{3}\right)$ for some $1 \leq j \leq s_{1}^{\prime}$. Since this is $\eta$-dense in red, there must be at least $\eta\left|V_{3}\right| / 2$ vertices in $V_{3}$ for which the red degree in $V_{1}^{i_{j}}$ is at least $\eta\left|V_{1}^{i_{j}}\right| / 2=\eta t_{1} / 2$. Indeed, otherwise the total number of red edges would be less than

$$
\frac{\eta}{2}\left|V_{3}\right|\left|V_{1}^{i_{j}}\right|+\frac{\eta}{2}\left|V_{3}\right|\left|V_{1}^{i_{j}}\right|=\eta\left|V_{3}\right|\left|V_{1}^{i_{j}}\right|
$$

a contradiction with the fact that $\left(V_{1}^{i_{j}}, V_{3}\right)$ is $\eta$-dense in red. Consider all the red neighborhoods of these vertices in $V_{1}^{i_{j}}$. Since there can be at most

$$
2^{t_{1}}=n^{\frac{\beta}{2 \log \frac{1}{\eta}}}
$$

such neighborhoods, by averaging and using (2) and (4) there must be a red neighborhood that appears for at least

$$
\frac{\frac{\eta}{2}\left|V_{3}\right|}{n^{\frac{\beta}{2 \log \frac{1}{\eta}}}} \geq \frac{\eta}{8} n^{1-\frac{\beta}{2 \log \frac{1}{\eta}}} \gg \frac{\eta}{2} t_{1}
$$

such vertices of $V_{3}$. This means that we can find a red complete balanced bipartite graph $K\left(t_{2}, t_{2}\right)$ in the red bipartite graph $\left(V_{1}^{i_{j}}, V_{3}\right)$, where $t_{2}=\eta t_{1} / 2$. We can proceed similarly for the red bipartite graph $\left(V_{2}^{i_{j}}, V_{3}\right)$. Thus the red complete balanced bipartite graph $K_{i j}\left(t_{1}, t_{1}\right)$ can be replaced with three red complete balanced bipartite graphs. We can proceed similarly for all $K_{i_{j}}\left(t_{1}, t_{1}\right), 1 \leq j \leq s_{1}^{\prime}$. This way we obtain a new red well-connected complete bipartite graph cover

$$
M_{2}=\left(K_{1}\left(t_{1}^{(2)}, t_{1}^{(2)}\right), K_{2}\left(t_{2}^{(2)}, t_{2}^{(2)}\right), \ldots, K_{s_{2}}\left(t_{s_{2}}^{(2)}, t_{s_{2}}^{(2)}\right)\right)
$$

such that

- $\left|M_{2}\right| \geq\left(1+\frac{\eta^{2}}{2}\right)\left|M_{1}\right|$, and

- $t_{1} \geq t_{i}^{(2)} \geq t_{2}=\eta t_{1} / 2$ for every $1 \leq i \leq s_{2}$.

Iterating this process $(j-1)$ times we get a new cover

$$
M_{j}=\left(K_{1}\left(t_{1}^{(j)}, t_{1}^{(j)}\right), K_{2}\left(t_{2}^{(j)}, t_{2}^{(j)}\right), \ldots, K_{s_{j}}\left(t_{s_{j}}^{(j)}, t_{s_{j}}^{(j)}\right)\right)
$$

such that

- $\left|M_{j}\right| \geq\left(1+\frac{\eta^{2}}{2}\right)^{j-1}\left|M_{1}\right|$, and

- $t_{1} \geq t_{i}^{(j)} \geq t_{j}=(\eta / 2)^{j-1} t_{1}$ for every $1 \leq i \leq s_{j}$.

This and (3) imply that if we could iterate this process $8 / \eta^{3}$ times, then we would get a red complete balanced bipartite graph cover of size at least $(2 / 3+2 \eta) n$, where for each $K_{i}\left(t_{i}, t_{i}\right)$ in the cover we would still have

$$
t_{i} \geq\left(\frac{\eta}{2}\right)^{\frac{8}{\eta^{3}}} t_{1}=\left(\frac{\eta}{2}\right)^{\frac{8}{\eta^{3}}} \frac{\beta}{2 \log \frac{1}{\eta}} \log n=c \log n
$$

and thus we would have Case 1 in Lemma 3.1. Indeed, using (3) in each iteration we increase the size of the cover by

$$
\frac{\eta^{2}}{2}\left|M_{1}\right| \geq \frac{\eta^{3}}{8} n
$$

and thus in at most $8 / \eta^{3}$ iterations we get a cover of size at least $(2 / 3+2 \eta) n$.

We may assume that this is not the case and after $(j-1)$ iterations for some $j<8 / \eta^{3}$ we get a cover $M_{j}$ as above for which (i) does not hold. In this case we will show that we can find directly a monochromatic well-connected complete bipartite graph cover of size at least $(2 / 3+2 \eta) n$ unless we are in the Extremal Coloring (Case 2). For simplicity we still use the notation $V_{1}, V_{2}, V_{3}$ for $M_{j}$ just as for $M_{1}$. Since (i) does not hold for $M_{j}$, for most of the complete bipartite graphs $K_{i}\left(t_{i}^{(j)}, t_{i}^{(j)}\right)=\left(V_{1}^{i}, V_{2}^{i}\right)$ (namely for complete bipartite graphs covering at least a $(1-\eta)$-fraction of the total size of $\left.M_{j}\right)$ the red density in one of the bipartite graphs $\left(V_{1}^{i}, V_{3}\right)$ and $\left(V_{2}^{i}, V_{3}\right)$ is less than $\eta$. By renaming we may assume that this is always the bipartite graph $\left(V_{2}^{i}, V_{3}\right)$. This means that indeed $\left(V_{2}, V_{3}\right)$ is $(1-2 \eta)$-dense in blue-only, as we wanted. However, this implies that

$$
\left|V_{3}\right|<\left(\frac{1}{3}+\alpha^{3}\right) n
$$

since otherwise using (2) we can easily find a blue well-connected complete balanced bipartite graph cover of size at least $(2 / 3+2 \eta) n$ by iteratively applying Lemma 2.1 in blue, first in the bipartite graph $\left(V_{2}, V_{3}\right)$ while we can, and then continuing 
inside $V_{3}$. Similarly if the blue density in the bipartite graph $\left(V_{1}, V_{2}\right)$ is at least $\alpha^{3}$, then again we can find a blue wellconnected complete balanced bipartite graph cover of size at least $(2 / 3+2 \eta) n$ by iteratively applying Lemma 2.1 in blue, first in the bipartite graph $\left(V_{1}, V_{2}\right)$, then in the bipartite graph $\left(V_{2}, V_{3}\right)$, and finally inside $V_{3}$. Thus we may assume that the bipartite graph $\left(V_{1}, V_{2}\right)$ is $\left(1-\alpha^{3}\right)$-dense in red-only.

Making progress towards the Extremal Coloring, next let us look at the red density inside $V_{2}$. Assume first that this density is at least $\alpha^{2}$. Similarly as above this implies that the bipartite graph $\left(V_{1}, V_{3}\right)$ is $\left(1-\alpha^{2}\right)$-dense in blue-only, since otherwise we can find again a red cover of size at least $(2 / 3+2 \eta) n$. Thus the bipartite graph $\left(V_{1} \cup V_{2}, V_{3}\right)$ is $\left(1-\alpha^{2}\right)$-dense in blue-only. This in turn implies that $V_{1} \cup V_{2}$ is $(1-\alpha)$-dense in red-only since otherwise we can find again a blue cover of size at least $(2 / 3+2 \eta) n$. This gives us the Extremal Coloring where $A=V_{1} \cup V_{2}, B=V_{3}, G_{1}$ is red and $G_{2}$ is blue (actually here in a somewhat stronger form since inside $B$ most of the edges are blue-only as well).

Hence we may assume that $V_{2}$ is $\left(1-\alpha^{2}\right)$-dense in blue-only. This implies again that $\left(V_{1}, V_{3}\right)$ is $(1-\alpha)$-dense in red-only and this gives us the Extremal Coloring again where $A=V_{2} \cup V_{3}, B=V_{1}, G_{1}$ is blue and $G_{2}$ is red. This finishes the proof of Lemma 3.1.

\section{Acknowledgements}

The first author's research is supported in part by OTKA Grant No. K68322. The second author's research was supported in part by the National Science Foundation under Grant No. DMS-0456401, by OTKA Grant No. K68322 and by a Janos Bolyai Research Scholarship. The third author's research was supported in part by the Ellentuck Fund.

\section{References}

[1] B. Bollobás, Extremal Graph Theory, Academic Press, London, 1978.

[2] B. Bollobás, P. Erdős, M. Simonovits, On the structure of edge graphs II, J. London Math. Soc. 12 (2) (1976) $219-224$

[3] L. Gerencsér, A. Gyárfás, On Ramsey-type problems, Ann. Univ. Sci. Budapest Eötvös Sect. Math. 10 (1967) 167-170.

[4] R.L. Graham, B.L. Rothschild, J.H. Spencer, Ramsey Theory, John Wiley \& Sons, New York, 1990.

[5] A. Gyárfás, M. Ruszinkó, G.N. Sárközy, E. Szemerédi, Three-color Ramsey numbers for paths, Combinatorica 27 (2007) $35-69$.

[6] A. Gyárfás, G.N. Sárközy, E. Szemerédi, Monochromatic Hamiltonian 3-tight Berge cycles in 2-colored 4-uniform hypergraphs (submitted for publication).

[7] Y. Kohayakawa, M. Simonovits, J. Skokan, The 3-colored Ramsey number of odd cycles, manuscript.

[8] P. Kővári, V.T. Sós, P. Turán, On a problem of Zarankiewicz, Colloq. Math. 3 (1954) 50-57.

[9] I. Levitt, G.N. Sárközy, E. Szemerédi, How to avoid using the regularity lemma; Pósa's conjecture revisited, Discrete Math. (in press).

[10] V. Nikiforov, R.H. Schelp, Cycles and stability, J. Combin. Theory Ser. B 98 (2008) 69-84.

[11] S.P. Radziszowski, Small Ramsey numbers, Electron. J. Combin. (2002) DS1.

[12] F.P. Ramsey, On a problem of formal logic, Proc. London Math. Soc., 2nd Ser. 30 (1930) 264-286.

[13] E. Szemerédi, Regular partitions of graphs, in: Colloques Internationaux C.N.R.S. № 260 - Problèmes Combinatoires et Théorie des Graphes, Orsay, 1976, pp. 399-401. 